\title{
Review on Corner Detection Algorithms
}

\author{
Xing-Fu Zhang, Yan-Bo Li, Jing-Feng Song \\ College of Computer Science and Technology, Heilongjiang Institute of Technology, Harbin, China \\ E-mail: portzhang@foxmail.com, 53399864@qq.com
}

\begin{abstract}
Corner detection algorithms are widely used in image recognition, image matching and edge detection. Accurately finding a corner in a image can greatly improves the algorithms accuracy of the above research areas. So many scientists and researchers have begun to the hot research field and have made much remarkable achievements. The paper introduced the theory of classic corner detection algorithm, including Harris algorithm, surf and sift algorithm, ORB algorithm, Susan algorithm and so on, And compared the difference of classic algorithms. Then, the recently outstanding algorithm and its application are introduced. Finally, the paper summarizes the research trends about corner detection algorithms.
\end{abstract}

\section{Keywords- corner detection; harris; sift; surf; orb}

\section{INTRODUCTION}

Image is made up of pixels, to accurately find the pixel features are the basis of many image and video algorithm. Corner points are more obvious change points relative to the surrounding pixel, and finding corner points are one of the important procedure of image feature detection. Corner points can represent the characteristics of the image to a certain extent. Most corner detection algorithm often choose a small area around the pixel point or pixel area, we call the area window. If the window is moving in all directions, a significant change is generated, we can think the pixel or pixel region is a corner. The accuracy of the corner detection, sensitivity to noise and the calculation speed are often used to evaluate the performance of the corner detection algorithm. Corner detection algorithm is widely used for edge detection, image matching and moving object detection. In this paper, the classical corner detection algorithm is described and summarized. And the recently research of the classical algorithms are summarized too. Finally, we point out the future research focus of the corner detection algorithm.

\section{CLASSIC CORNER DETECTION ALGORITHMS}

\section{A. SIFT and SURF Algorithms}

The main flow of the SIFT algorithm is shown in Figure 1. For an original image, the use of SIFT algorithm for image feature extraction, and feature description. And then for a test image using a similar process, get the feature description of the test image. At last, the feature points of two images are matched.

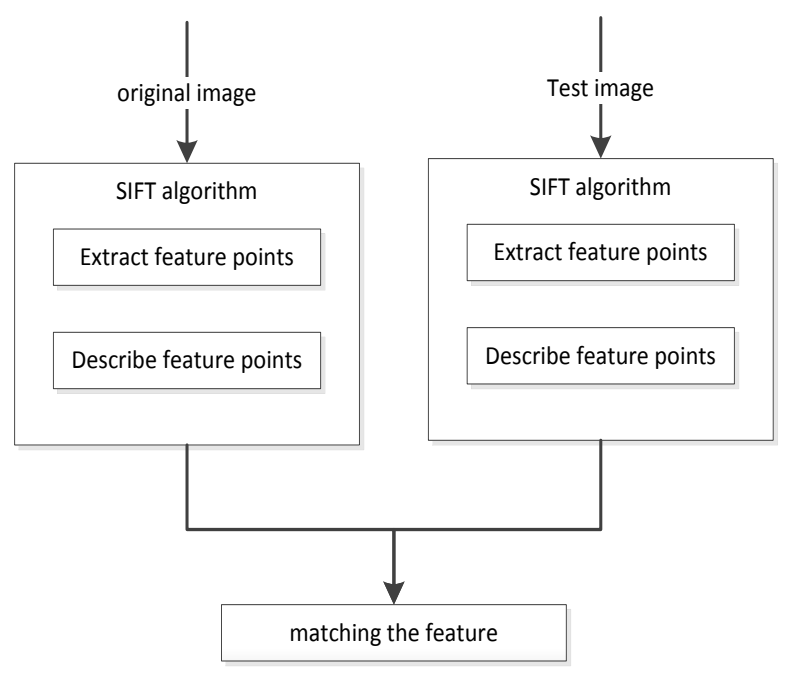

Figure 1. Flow chart of SIFT algorithm.

First, the SIFT algorithm [1] needs to solve the problem of scale change. Koenderink [2] and Lindeberg [3] have proved that the only possible Kernel function is the Gauss function. So we can use the following functions to define the scale space function.

$$
L(x, y, \delta)=G(x, y, \delta) * I(x, y)
$$

The asterisk represents convolution, the I represents the gray function of an image, and the $\mathrm{G}$ represents Gauss function.

Secondly, the SIFT algorithm want to solve the problem of rotation. It selects a main direction for each feature point. When we want to match the image, the first step is to rotate the image to be matched with the main direction. SIFT used the Histogram algorithm to find the main direction. The calculation formula is as follows.

$$
\theta(\mathrm{x}, \mathrm{y})=\tan ^{-1}\left(\frac{L(x, y+1)-L(x, y-1)}{L(x+1, y)-L(x-1, y)}\right)
$$

Finally, SIFT algorithm describes the feature points and uses the Euclidean distance as the similarity measure. SIFT algorithm is a feature-based matching algorithm, which has the advantage of scale and rotation invariant Compared with other features matching algorithm. The algorithm is still outstanding when the image exists affine distortion, the change of angle and so on. The disadvantage of SIFT 
algorithm is that the computation is very large and not suitable for real-time detection system.

SURF algorithm [4] is the improvement of SIFT algorithm, which uses the box filter to replace the two order Gauss filter and uses the haar wavelet instead of the histogram. SURF algorithm is no longer required for image resampling, so the algorithm reduces the time and memory usage, greatly improving the feature point extraction speed.

\section{B. Harris Algorithm}

Harris algorithm [5] determines the principle of corner points in an image. That is to say, the pixel values in the window are unchanged When a small window is moved outside the corner along any direction. But the pixel values will change significantly when a small window is moved inside the corner. Set window translation $[\mathrm{u}, \mathrm{v}]$ and the gray level of the window is $\mathrm{E}(\mathrm{u}, \mathrm{v})$, then,

$$
E(u, v)=\sum_{x, y} w(x, y)[I(x+u, y+v)-I(x, y)]^{2}
$$

Where, $w(x, y)$ is a window function and $I(x, y)$ is image gray of the window. $I(x+u, y+v)$ is the image gray after moving the window. Usually we use a rectangular function or Gaussian function as a window function. Eigenvalues obtained by matrix calculation can determine whether a point corner.

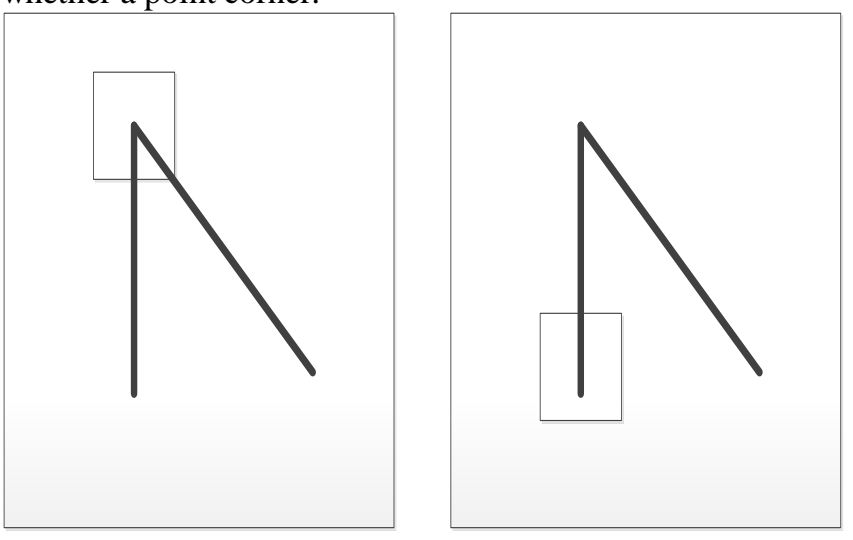

Figure 2. Harris algorithm window sliding.

As shown in Figure 2, the Harris algorithm mainly depends on window sliding to calculate the pixel value in the window. Similar to figure 2 shown on the left, when the window is moving near the corner, the pixel value in the window will change greatly. Similar to figure 2 shown on the right, when the window is moving near the edge, the pixel value in the window will change smaller.

\section{SUSAN Algorithm}

SUSAN algorithm [6] was presented in 1995 by S.M.Smith and J.M.Brady, of the University of Oxford in the UK. The algorithm defines a small radius circle as a template. Then we slide the template on the image in a certain order. If the sliding to a region of the image to meet that each pixel's gray value in the template is similar to the center gray values of the image. We call the region USAN and call the center pixel kernel. The number of pixels in the same or similar to the kernel gray value is called the template area. When the kernel is just located at the corner, the USAN area is the smallest. SUSAN algorithm has the following characteristics: The algorithm does not use gradient and other mathematical concepts, so the calculation speed is fast, which is suitable for real time applications. First, the algorithm does not use the gradient and other mathematical concepts, so the calculation speed is very fast and suitable for real-time applications. Secondly, the algorithm has good robustness and is not sensitive to single point noise. Finally, the algorithm can be used for edge detection and corner detection, but it is more suitable for corner detection.

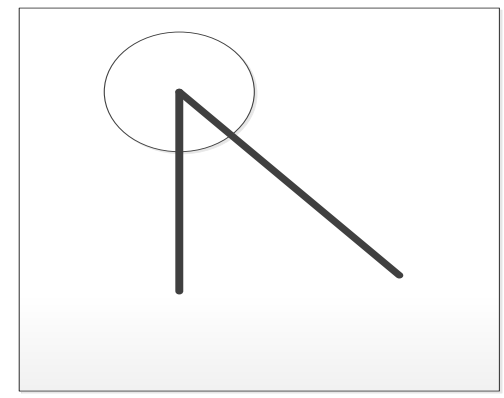

Figure 3. Circular sliding window of SUSAN algorithm.

Different from the other algorithms, the SUSAN algorithm uses a circular template to slide along a specific direction in the image. As shown in Figure3. If the difference between the gray level of the pixel in the template and the center of the template is less than the specified threshold value, it is considered that the point and the template center pixel have similar gray level. All regions that satisfy such conditions are called Univalue Segment Assimilating Nucleus. When the circular template is completely in the image or background, the area of Univalue Segment Assimilating Nucleus is the largest. When the circular template is moved to the edge of the image, the Univalue Segment Assimilating Nucleus region gradually becomes smaller. When the center of the circular template is at the corner, the Univalue Segment Assimilating Nucleus region is the smallest. No matter to the edge or corner, the SUSAN algorithm can achieve better results. The SUSAN algorithm does not involve the calculation of the gradient, so the algorithm is very robust to noise.

\section{ORB Algorithm}

ORB algorithm [7] is a feature detection and feature description algorithm. It is said to be the fastest corner detection algorithm. The ORB algorithm can match the object with the change of the plane angle or the proportion. ORB algorithm mainly involves FAST and BRIEF algorithms. It uses the FAST algorithm to find the corner points. That is to say, if the gray value of the neighborhoods of a certain pixel is most more than or less than the gray value of the pixel, the point is defined as a corner point. The BRIEF algorithm is the description of the corner, and describes the characteristics of the corner. When the 
similarity between the features of a point in the image and the known points is greater than a certain threshold, we believe that the two points are similar. ORB algorithm improves the FAST and BRIEF algorithms and the improved algorithm are called oFAST and rBRIEF.

FAST and oFAST algorithms. Take a circle in an image, the center is $\mathrm{p}$ and the radius is $\mathrm{r}$. Select $\mathrm{n}$ pixels on the circle (usually the value of $n$ is 12). There are the following fomula.

$$
C R F=\left\{\begin{array}{l}
1, \text { if }\left|I_{p}-I_{k}\right|>t \\
0, \text { else }
\end{array}\right.
$$

Where, $I_{p}$ is the pixel value of the center pixel. $I_{k}$ is the pixel value of any point on the circle. $t$ is a very small threshold. If the CRF value of more than 12 points of the 16 points on the circle is 1 , we think the $\mathrm{p}$ as a corner point.

oFAST algorithm introduces the concept of main direction on the basis of FAST algorithm. First, oFAST used the FAST algorithm to detect a corner. Second, oFAST algorithm computes a centroid. Finally, the main direction of the image patch is determined by the connection line of the center point and the centroid. Where the centroid mainly obtains by computing the $\mathrm{n}$ order moment.

BRIEF and rBRIEF algorithms. BRIEF algorithm. A number of pairs of points are randomly selected to form an image block near the feature point. Binarization processing to image blocks and to form a binary string. That is to say, for a point $\mathrm{p}$ in an image, we describe it using the relationship of pairs of points near it. Take two points from two images respectively. If two points' description is the same or similar, we can say that the two points are similar. For a point in an image, we randomly selected two points $\mathrm{x}$ and $\mathrm{y}$. The relationship between $\mathrm{x}$ and $\mathrm{y}$ is used as a component of the feature vector.

$$
\tau(p ; x, y)=\left\{\begin{array}{l}
1, \text { if } p(x)<p(y) \\
0, \text { else }
\end{array}\right.
$$

For each pair of points, we compute the value 0 or 1 by the above formula. $\mathrm{N}$ pair of points according to a certain sequence consisting of a n-dimensional vector. We call the ndimensional vector description describer of $p$. BRIEF algorithm often uses Hamming distance computing the similarity.

rBRIEF algorithm. An n-dimensional random vector, each component consists of 0 or 1 , so the expectation should be 0.5 . In order to obtain a better ability to distinguish, we hope that the random vector of the variance as large as possible. RBRIEF algorithm first uses BRIEF steered algorithm, then use the greedy algorithm to select the large variance of the random pair of points. In the use of the algorithm, some noise reduction algorithms are often used to increase the accuracy of the ORB algorithm. Generally, the image is filtered by Gauss filter, filtering the outliers in the image, so that the image is relatively smooth. We can also use a small image block instead of a point, so that the algorithm is more robust.

\section{ADVANCES IN CORNER DETECTION ALGORITHMS}

Corner detection algorithm has great research values, so many scientists are actively involved in the study of the algorithm. There are mainly two aspects of the study. On the one hand, it is to study the improvement of the relevant corner algorithm, which improves the various aspects performance of the algorithm. On the other hand, it is to solve the practical problems by applying the relevant corner detection algorithm to the practical application.

Zhuo Li et al. in 2015 [8], the corner detection algorithm is applied to the pornographic image detection. We must detect the target image quickly and accurately for the application of pornographic image detection. Corner detection algorithm is used in this paper. Since the application needs to be detected very quickly, the paper ORB algorithm is used as the corner detection algorithm in this paper. Guo Yongfang et al. in 2012 [9], they use Harris corner detection algorithm and Trajkovic fast detection algorithm for feature points matching, and achieved good results. Zhu Hongbo et al. in 2011 [10], the authors improve the SIFT algorithm. They fuse local statistical features and corner features in the algorithm, and then use the improved algorithm to image annotation, which greatly improves the accuracy and computational speed. Bo Yu et al. in 2014 [11], they use the Harris corner detection algorithm to detect the region, increasing the robustness and effectiveness of the detection results. Sun Cheol Bae et al. in 2002 [12], in this paper, two oriented cross operators called crosses as oriented pair(COP) are used. The algorithm is improved in the aspects of fast detection, accurate detection and noise immunity compared with previous algorithms. Pablo F. Alcantarilla et al. in 2013 [13], G-SURF algorithm is proposed in this paper, which is based on second-order multiscale gauge derivatives. In the end of the paper, some complex background images are selected to compare the algorithm and some classical algorithms. The results show that the performance of GSURF algorithm is better than that of the traditional SURF algorithm.

In addition to the above, there are a lot of scholars research and improve the corresponding algorithms, and continue to apply the algorithms in various aspects of the image processing, and achieved many good results. In the coming period, the research on the algorithm of corner detection will always be a hot research topic.

\section{COMPARISON OF SEVERAL ALGORITHMS}

Corner detection algorithm is the key problem of local feature description. It plays a very important role in the subsequent feature extraction and feature description algorithm. This series of corner matching algorithm has been widely used in image classification, image recognition, image orientation and other fields. The traditional feature extraction methods are not robust enough, and often have little information on spatial information. But corner detection 
algorithm can be used to record the change of spatial information. So the corner detection algorithm often has a good robustness under the condition of the existence of occlusion and other conditions.

Harris algorithm is a basic corner detection algorithm, the principle is simple, easy to understand. The main method is to use a small sliding window, sliding along a certain direction. Only the window is sliding near the corner point, the pixel gray will be a big change. While the window is sliding near the non-corner point, the pixel gray change is very small. Harris algorithm is simple, easy to understand, and the calculation of this algorithm is very small, so it has a lot of practical. But the algorithm also has a major drawback, that is, does not have scale invariance.

SIFT algorithm was proposed by D.Lowe as early as 1999. The proposed algorithm greatly improves the performance of corner detection. SIFT algorithm is invariant to scale, rotation, and changes in a certain angle of view and illumination changes. SURF algorithm is an improvement of SIFT algorithm. The algorithm uses Haar wavelet to approximate the gradient and uses the integral image to quickly calculate. In the best case, the speed of the surf algorithm can be 6 times faster than the SIFT algorithm. The essence of SIFT algorithm is to find the key feature points in different scale space. The robustness of the feature points obtained using the SIFT algorithm is better. The feature points obtained by this method will not be changed because of the light, affine transformation, noise and partial occlusion and so on. Sift and Surf algorithm mainly uses the Henssian matrix to get the local extremum of the image. But in the process of seeking the main direction is too dependent on the local area of the pixel gradient direction. This is likely to cause the main direction is not accurate. The main direction is the core of this algorithm, if the main direction is not accurate, then the subsequent feature extraction is also easy to become inaccurate. Then the performance of the whole algorithm will be greatly reduced. The main differences between the SIFT algorithm and the SURF algorithm are as follows. First, the image preprocessing methods are different. SITF algorithm will first expand the image in all directions by two times, and the SURF algorithm is to compute the integral image directly. Second, the feature vector dimensions of the second algorithms are different. SIFT algorithm is eight directions, a total of 128 dimensions and SURF algorithm is four directions, a total of 64 dimensions. Third, the method of noise point treatment is different. SIFT algorithm using RANSAC algorithm to remove the noise points and SURF algorithm does not need to remove the noise points.

BRIEF algorithm use gray image size of the local neighborhood of random point to establish local image descriptors. The computing speed of the feature description operator is very fast by using the BRIEF algorithm. BRIEF algorithm provides a more efficient way to calculate the binary string. That is to say, the BRIEF algorithm use Hamming distance to match the feature points. We need to be aware that the BRIEF algorithm provides us with a feature description algorithm, which does not provide a feature extraction method.
ORB algorithm mainly uses the FAST algorithm to detect the feature points, and then use the BRIEF algorithm to feature description. ORB algorithm has the characteristics of rotation invariance. The rotation invariance is achieved mainly by the gray centroid method. The method can find the centroid of the image slices, so as to determine the main direction of the image. When two images are matched, one image is rotated in order to ensure that the two images are the same as the main direction. This method is realized by the so-called rotation independent. ORB algorithm to select the BRIEF algorithm for feature point description. But we all know that the BRIEF algorithm itself does not have the rotation invariant property. So ORB algorithm to improve the BRIEF algorithm, is called the BREIF Steer algorithm. The rotation invariant property is achieved.

\section{CONCLUSION}

This paper mainly summarizes the classical corner detection algorithm, including SIFT algorithm, SURF algorithm, Harris algorithm, SUSAN algorithm and ORB algorithm, etc., and then the recently improved algorithms and its application are introduced. The paper also makes a detailed comparative analysis of the advantages and disadvantages of several algorithms mentioned in the paper. Finally, the paper points out that the corner detection is still a research hot spot in the future. With the gradual rise of image recognition, image classification, image location and other applications, the study of image feature extraction and image feature description will also in the development of the peak. Corner as an important feature of the image, it will receive more and more attention of researchers. Corner detection is also bound to attract more and more researchers' attention.

\section{ACKNOWLEDGMENT}

The research work was supported by Science and technology research project of Education Department of Heilongjiang Province (12541661)

[1] D. G. Lowe. "Distinctive image features from scale-invariant keypoints, " International Journal of Computer Vision, vol. 60,2004, pp. 91-110.

[2] JJ Koenderink. "The structure of images," Biological Cybernetics, vol. 50,1984, pp. 363-370.

[3] T Lindeberg. "Scale-space theory in computer vision," Springer Berlin, vol. 256, 1994, pp. 349-382.

[4] H. Bay, T Tuytelaars, and L. Van Gool. "Surf: Speeded up robust features," Computer Vision and Image Understanding, vol. 101, 2006, pp. 404-417.

[5] C.Harris, M.Stephens. "A Combined Corner and Edge Detector," In:Fourth Alvey Vision Conference, IEEE Press, 1998, pp.147-151.

[6] SMITH S. M, BRADY J. M. SUSAN. "A new approach to low level image processing," International Journal of Computer Vision, vol. 23,1997, pp. 45-78.

[7] E Rublee, V Rabaud, K Konolige. "G Bradski. ORB: An efficient alternative to SIFT or SURF," In: ICCV Barcelona IEEE Press, 2011, pp.2564-2571.

[8] Li Zhuo, Zhen Geng, Jing Zhang, Xiao guang Li. "ORB feature based web pornographic image recognition,” Neurocomputing, In Press.

[9] Guo Yongfang, Yu Ming, Sun Yicai. "Study on an Improved Robust Algorithm for Feature Point," Physics Procedia. Vol.33, 2012,pp. 1810-1816. 
[10] Zhu Hongbo, Xu Xuejun, Wang Jing, Chen Xuesong, Jiang Shaohua. "A Rapid Automatic Image Registration Method Based on Improved SIFT," Procedia Environmental Sciences, vol. 11,2011,pp. 85-91.

[11] Bo Yu, Li Wang, Zheng Niu. "A novel algorithm in buildings/shadow detection based on Harris detector," Optik - International Journal for Light and Electron Optics, vol. 125,2014, pp. 741-744.
[12] Sun Cheol Bae, In So Kweon, Choong Don Yoo. "COP: a new corner detector," Pattern Recognition Letters, vol. 23, 2012, pp. 1349-1360.

[13] Pablo F. Alcantarilla, Luis M. Bergasa, Andrew J. Davison. "GaugeSURF descriptors," Image and Vision Computing, vol. 31,2013, pp 103-116. 